\title{
L'hydrologie de la crue de 1910 et autres grandes crues du bassin de la Seine
}

\author{
par R. Marti, Th. Lepelletier
}

Hydratec

\section{I ם GENÈSE ET DYNAMIQUE DE LA CRUE DE JANVIER 1910}

\subsection{Principales caractéristiques géomorphologiques du bassin de la Seine en amont de Paris}

Le bassin de la Seine en amont de Paris occupe une superficie totale de $44000 \mathrm{~km}^{2}$. Les bassins versants associés peuvent être regroupés en trois catégories :

- les bassins imperméabilisés et (ou) à forte pente répartis sur la périphérie amont et comprenant principalement : 1'Yonne, l'Armançon, le Serein, la Haute-Seine, l'Aube, la Haute-Marne, la Saulx,

- la zone perméable du centre occupant la vallée de la Marne entre Frignicourt et Epernay, la Petite Seine et l'Yonne à l'aval d'Auxerre,

- les terrains variés de la région Ile-de-France avec des vallées à fort pouvoir de ruissellement (les Morin, l'Yerres, le Loing), des vallées peu productives (Essonne, Ourcq) et de nombreuses zones péri-urbaines.

Lors de la génération des grande crues, $90 \%$ des apports de ruissellement proviennent de la zone périphérique amont, qui couvre $40 \%$ du territoire.

On peut considérer que les principales ondes de crue, dont la concomitance détermine le maximum à Paris, sont formées :

- à la confluence Seine-Aube pour la Petite Seine,

- à Joigny pour l'Yonne,

- à Chalons pour la Marne.

Dans le cas d'épisodes pluvieux multiples, les apports en provenance des affluents rapides d'Ile-de-France peuvent influer sur les niveaux atteints à Paris.
De cette rapide présentation, il ressort que l'hydrologie de la crue de 1910 est bien cernée par la connaissance des apports en provenance des sous-bassins :

— de l'Yonne, de l'Armançon et du Serein,

- de la Haute Marne,

— de la Haute Seine et de l'Aube.

\section{- 1.2 Contexte climatique}

Les évolutions comparées des hauteurs d'eau de la Haute Seine, de la Marne, de l'Yonne amont et de la pluviométrie cumulée entre août et décembre 1909 font apparaître :

- une pluviométrie très abondante sur ces trois vallées durant le dernier trimestre 1909 , avec une hauteur totale de précipitation d'environ $450 \mathrm{~mm}$, soit plus du double de la valeur moyenne enregistrée habituellement durant cette période de l'année,

- une réaction aux épisodes pluvieux de cette période très atténuée sur la Marne et la Haute Seine, plus sensible sur l'Yonne et ses affluents,

- une réaction très brutale pour les trois vallées à l'épisode pluvieux du 15 au 22 janvier qui n'a pourtant pas été beaucoup plus marqué en hauteur cumulée que certains épisodes précédents.

La crue de janvier 1910 a donc bénéficié de conditions de sols saturés. Bien qu'il n'y ait pas eu de pluies préparatoires entre le ler et le 10 janvier, les nappes étaient entièrement rechargées et les conditions de ruissellement très actives.

\subsection{Pluviométrie}

La crue de janvier 1910 a été engendrée par l'épisode pluvieux généralisé du 15 au 20 janvier sur le bassin de la Seine en amont de Paris, suivi d'un deuxième épisode plu-

After describing qualitative features associated with the January 1910 flood event, this paper provides associated flow rates and comparison with other significant flood events during XXè century. These hydrological results are the basis for inundation risk analysis in today development conditions. 
vio-neigeux du 22 au 26 janvier. On note quelques précipitations entre le 10 et le 15 janvier, et aucune précipitation significative avant le 10 janvier.

Un troisième épisode pluvieux du 5 au 10 février génère une nouvelle pointe de crue atténuée, suivie d'un quatrième épisode du 20 au 28 février.

L'épisode du 15 au 20 a été extrêmement violent les 18 et 19 janvier, spécialement sur le Morvan et le Plateau de Langres.

On peut noter le fort gradient spatial de la pluviométrie sur l'Yonne, qui augmente significativement avec l'altitude ; les précipitations les plus fortes se sont produites aux Settons, avec une hauteur cumulée de $150 \mathrm{~mm}$ les 18 et 19 janvier.

\section{- 1.4 Genèse de la crue}

Les graphes de la figure 1 reproduisent la pluviométrie moyenne sur trois bassins versants amont et les relevés de l'échelle de crue à l'exutoire aval de chaque bassin : tous les bassins versants ont brutalement réagi. Cette soudaineté de la montée des eaux est attestée par les rapports des ingénieurs du SNS qui ont relaté l'événement vécu de 1910,
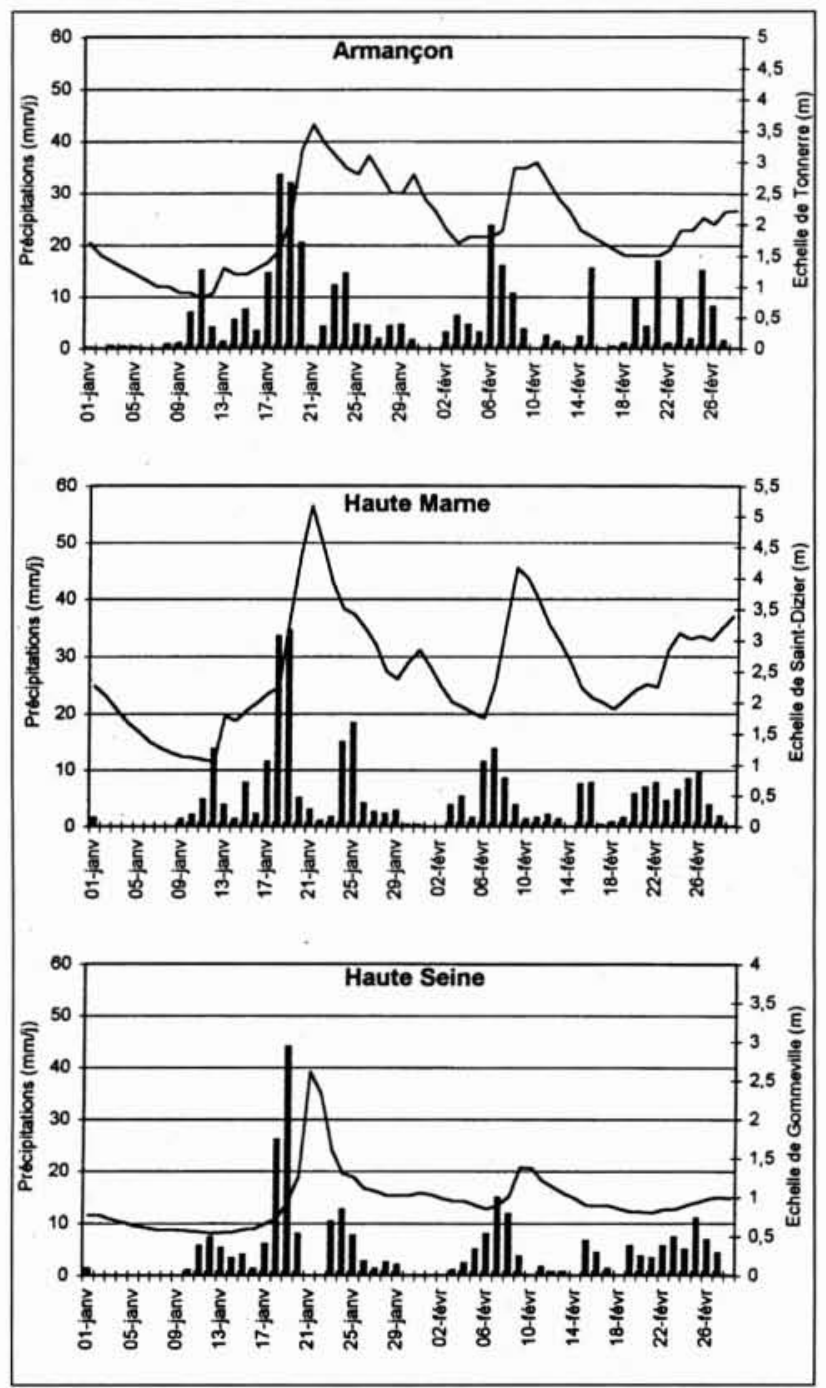

1. Hauteurs journalières de précipitations et limnigrammes relevés sur les bassins versants amont. notamment sur les bassins de la Haute Seine (à Troyes) et sur le Loing (à Montargis) [1]. La crue de 1910 est décrite comme ne ressemblant à aucun autre événement vécu, de par la violence des écoulements observés. Les temps de concentration des grands bassins producteurs de ruissellement étaient pour la crue de 1910 raccourcis de 24 heures en moyenne par rapport à ce qui est constaté habituellement.

\subsection{Dynamique de la crue}

Le mécanisme de la crue et l'analyse des niveaux atteints aux différentes stations du bassin ont été décrits par la Commission des Inondations créée après la crue de 1910 et qui a remis son rapport le 7 avril 1910.

Le caractère exceptionnel de cette crue se révèle à l'examen des relevés des échelles limnimétriques de la figure 2 : - il existe un décalage entre le temps d'arrivée de la pointe de crue de l'Yonne et celle de la Marne à Paris, de l'ordre de 5 - 6 jours, soit environ l'intervalle de temps séparant les épisodes pluvieux 1 et 2 ,

- sur l'Yonne, la succession des deux épisodes pluvieux critiques 1 et 2 maintient le niveau des hautes eaux après la première pointe,

- le maximum à Montereau est formé par la superposition de la première pointe de la Petite Seine (S1) et de la seconde pointe de l'Yonne (Y2). Ce maximum est aggravé à Melun par le passage de la seconde pointe du Loing (L2),

- sur la Marne en aval de Meaux, il y a simultanéité de la première pointe de crue de la Marne amont (M1) et de la seconde pointe marquée du Grand Morin (G2),

- enfin le pic enregistré à Paris le 28 janvier résulte de la coïncidence parfaite des ondes de crue en provenance des vallées lentes de la Marne (M1), de la Petite Seine (S1) et des vallées rapides de l'Yonne (Y2), des Morins (G2) et du Loing (L2).

\section{II — RECONSTITUTION DE L'HYDROLOGIE}

\section{- 2.1 Méthodologie}

L'absence de données hydrométriques pour les stations de l'amont a conduit à élaborer une méthode de génération des apports de ruissellement à partir de la pluviométrie par une double transformation :

- calcul de la pluie efficace à partir de la lame d'eau brute moyenne précipitée sur chaque bassin versant,

- calcul des hydrogrammes ruisselés à partir de la pluie efficace via une fonction de transfert affectée au bassin versant.

Les fonctions de transfert ont été établies sur les 10 bassins versants les plus productifs, à partir d'un calage réalisé sur des crues postérieures à 1980 [2].

Les apports des autres bassins versants ont été déduits par corrélation avec les hydrogrammes générés par fonction de transfert ou par simple corrélation avec la pluie : une soixantaine d'apports ont ainsi été reconstitués jusqu'à Mantes.

Dans les conditions de sols saturés, les coefficients de ruissellement varient suivant les bassins, de 0,5 à 0,8 . Ces conditions étaient réunies pour l'épisode du 15 au 22 janvier.

On a par ailleurs tenté, à partir des hauteurs relevées aux échelles, de bâtir les hydrogrammes de crue aux stations intermédiaires à partir des jaugeages effectués par le SNS durant la période 1870 - 1910 [3], en particulier aux stations de : 

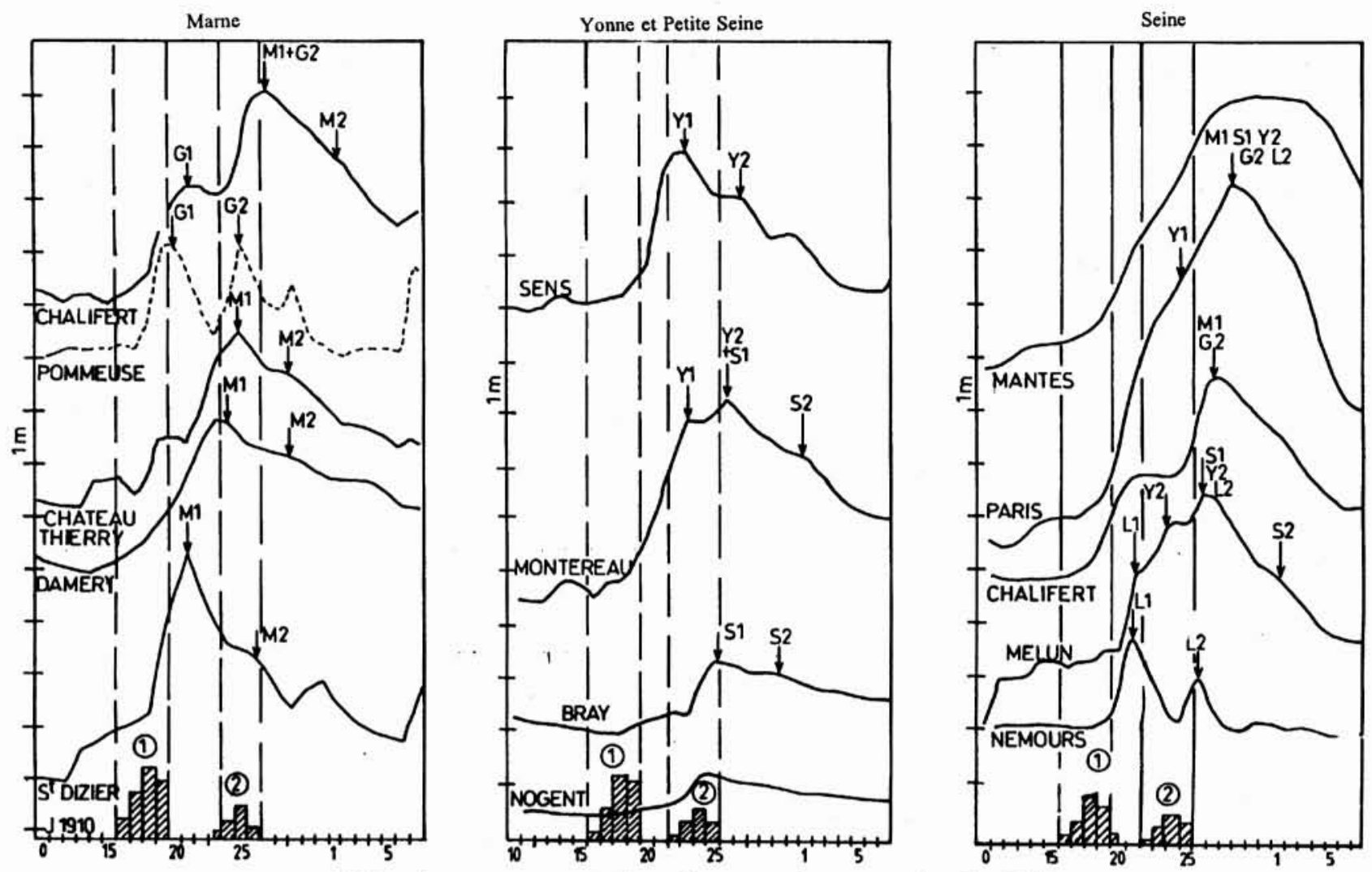

2. Limnigrammes aux stations d'annonce de crue en janvier 1910.

- Sens (Yonne),

- Chalifert (Marne),

- Melun (Seine amont),

- Paris Austerlitz,

- Mantes (Seine aval),

- Venette (Oise).

L'hydrologie élaborée à partir de la pluie a été ajustée par itérations successives à l'aide du modèle de propagation Seine - SBR, exploité par l'IIBRBS pour ses besoins propres de gestion : ce modèle simule la propagation d'une crue le long des vallées de l'Yonne, de la Marne et de la Seine, depuis l'amont des barrages réservoirs actuels jusqu'à Paris, à partir d'une hydrologie prédéterminée. Le travail de reconstitution de l'hydrologie a donc consisté, à chaque étape à :

- générer les apports à partir de la pluviométrie,

- propager les apports à l'aide du modèle hydraulique et comparer les formes des hydrogrammes calculés aux stations intermédiaires avec ceux déduits à partir des hauteurs d'échelles.

\section{- 2.2 Validation}

La figure 3 regroupe les hydrogrammes obtenus aux stations intermédiaires, à partir des hauteurs d'échelles d'une part, de la pluviométrie et le modèle de propagation d'autre part. Elle fait apparaître quelques distorsions, ce qui n'est pas étonnant compte tenu des imprécisions inhérentes à chaque approche. Néanmoins les débits de pointe et les instants de passage du maximum concordent plutôt bien.

Il convient de souligner que les débits propagés avec le modèle Seine-SBR intègrent la situation hydraulique actuelle

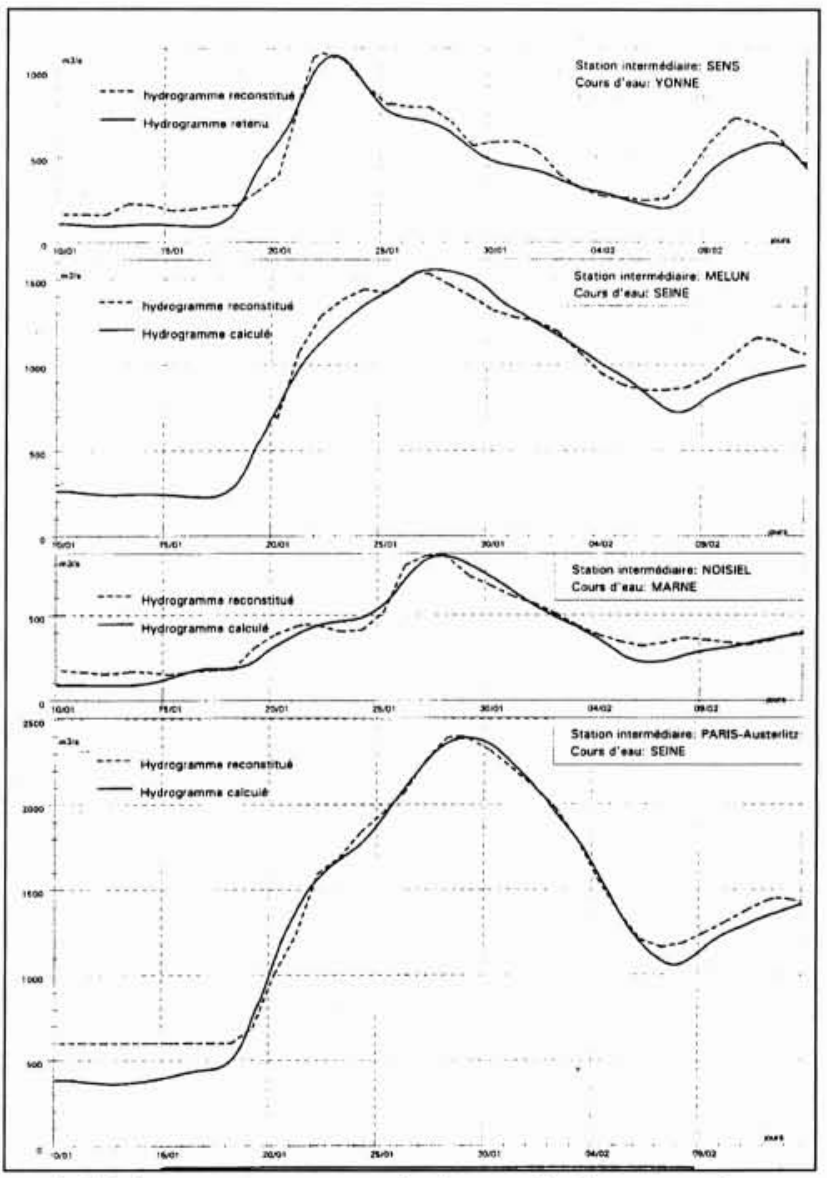

3. Hydrogrammes reconstitués et calculs aux stations intermédiaires pour la crue de janvier 1910. 
Tableau 1. - Stations amont du bassin.

\begin{tabular}{|c|c|c|}
\hline Station & Cours d'eau & $\begin{array}{c}\text { Débit maximum } \\
\mathrm{m}^{3} / \mathrm{s}\end{array}$ \\
\hline Gurgy & Yonne & 450 \\
Briennon & Armançon & 480 \\
Chablis & Serein & 180 \\
Bar-sur-Seine & Haute Seine & 350 \\
Troyes & Haute Seine & 450 \\
Bar-sur-Aube & Aube & 320 \\
Arcis & Aube & 380 \\
St Dizier & Haute Marne & 450 \\
Vitry & Saulx & 300 \\
Episy & Loing & 330 \\
\hline
\end{tabular}

Tableau 2. - Stations intermédiaires et aval.

\begin{tabular}{|c|c|c|}
\hline Station & Cours d'eau & $\begin{array}{c}\text { Débit maximum } \\
\mathrm{m}^{3} / \mathrm{s}\end{array}$ \\
\hline Marolles & Petite Seine & 680 \\
Sens & Yonne aval & 1100 \\
Melun & Seine amont & 1600 \\
Chalons & Marne amont & 920 \\
Chalifert & Marne aval & 860 \\
Paris & Seine moyenne & 2400 \\
Mantes & Seine aval & 2770 \\
\hline
\end{tabular}

qui diffère sensiblement des conditions de l'époque ; cependant la comparaison obtenue tend à démontrer que les modifications apportées aux lits mineur et majeur depuis 1910 ont peu influé sur les caractéristiques de propagation de la crue de Janvier 1910 compte tenu de son caractère très largement débordant le long de toutes les grandes vallées.

\section{III — DÉBITS MAXIMUM DE CRUE}

L'approche suivie conduit à estimer les débits de pointe suivants pour la crue de janvier 1910 (tableaux 1,2).

Cette crue a été réellement exceptionnelle sur l'Yonne, elle correspond au plus fort débit jamais observé.

La très forte pointe de crue à Paris provient de la succession, à cinq jours d'intervalle, des épisodes pluvieux (1) et (2) et des concomitances de pointes de crue qui en ont découlé.

La crue de janvier 1910 était modérée sur l'Oise (Qmax = $460 \mathrm{~m} 3 / \mathrm{s}$ à Venette) et n'a donc pas constitué un facteur d'aggravation sur la Seine aval.

Il est à noter que les jaugeages effectués sur la Seine aval par le SNS [5] durant l'épisode de crue de 1910, font état d'un débit au Pont de Bezons de $2700 \mathrm{~m}^{3} / \mathrm{s}$, soit $300 \mathrm{~m}^{3} / \mathrm{s}$ de plus que ce qui est retenu aujourd'hui.

Il s'avère après examen que les méthodes de jaugeages mises en œuvre à l'époque présentent un certain nombre de lacunes et d'imprécisions pouvant fort bien expliquer les fortes valeurs obtenues. On retiendra donc le chiffre de $2400 \mathrm{~m}^{3} / \mathrm{s}$ comme une estimation réaliste du débit de pointe à Paris.

\section{IV $\square$ COMPARAISON AVEC LES DEUX AUTRES GRANDES CRUES DE JANVIER 1924 ET JANVIER 1955}

\subsection{Crue de janvier 1924}

La documentation sur la crue de janvier 1924 provient des archives de la DIREN. Compte tenu de l'importance historique de cette crue, un travail de reconstitution similaire à celle de janvier 1910 a pu être entrepris et a fait l'objet d'un rapport spécifique [6].

Cette crue a une typologie très voisine de celle de 1910 : - automne particulièrement pluvieux avec une hauteur totale de précipitation de $400 \mathrm{~mm}$ en 3 mois, soit plus de deux fois la moyenne atteinte durant cette période,

- installation d'une zone dépressionnaire généralisée à l'ensemble du bassin en amont de Paris, avec trois trains successifs de précipitations d'une semaine chacun, espacés de 2 ou 3 jours :

- un épisode préparatoire, peu productif en ruissellement,

- un épisode intense fournissant une hauteur de précipitation moyenne de $100 \mathrm{~mm}$ sur 5 à 7 jours, homogène sur l'ensemble des bassins,

— un second épisode, se traduisant par une seconde pointe distincte sur l'Yonne,

- formation des 3 principales ondes de crue sur l'Yonne, la Marne et la Petite Seine, se combinant à Paris pour produire un débit maximum de $2100 \mathrm{~m}^{3} / \mathrm{s}$, le maximum à Paris coïncidant avec le passage des ondes de crue de la Marne, de la Petite Seine et de la seconde pointe de l'Yonne.

- absence de crue significative sur l'Oise (en amont de la zone de remous de la Seine) ; en fait, et c'est une chance pour la Seine aval, les crues de l'Oise et de la Seine sont rarement simultanées en raison de l'indépendance géographique des dépressions qui les génèrent : il n'existe pas d'exemple de pluviométrie qui intéresse une largeur de bande de $500 \mathrm{~km}$ Nord/Sud, couvrant l'ensemble du bassin de la Seine.

\subsection{Crue de janvier 1955}

La crue de janvier 1955 se démarque des deux autres événements, sous deux aspects :

- l'automne 1954 n'a pas été particulièrement pluvieux mais l'épisode qui a déclenché la grande crue de janvier s'est produit sur un sol gelé, avec des conditions de ruissellement similaires à celles d'un sol saturé d'eau,

- le second épisode pluvieux a été très peu fourni, voire inexistant : la pointe de crue à Paris n'a donc pratiquement pas été appuyée par la seconde pointe de l'Yonne.

\subsection{Débits maxima atteints}

Le diagramme de la figure 4 récapitule les débits maxi reconstitués pour chaque crue aux stations intermédiaires des bassins et les hauteurs d'eau relevées correspondantes.

- les trois crues ont été d'égale importance sur la Seine amont, - les crues de janvier 1910 et janvier 1955 ont été très proches sur la Marne, la crue de 1924 un peu moins soutenue sur cette vallée,

- les crues de 1955 et 1924 sont d'égale importance sur l'Yonne, celle de janvier 1910 a été encore plus marquée du 


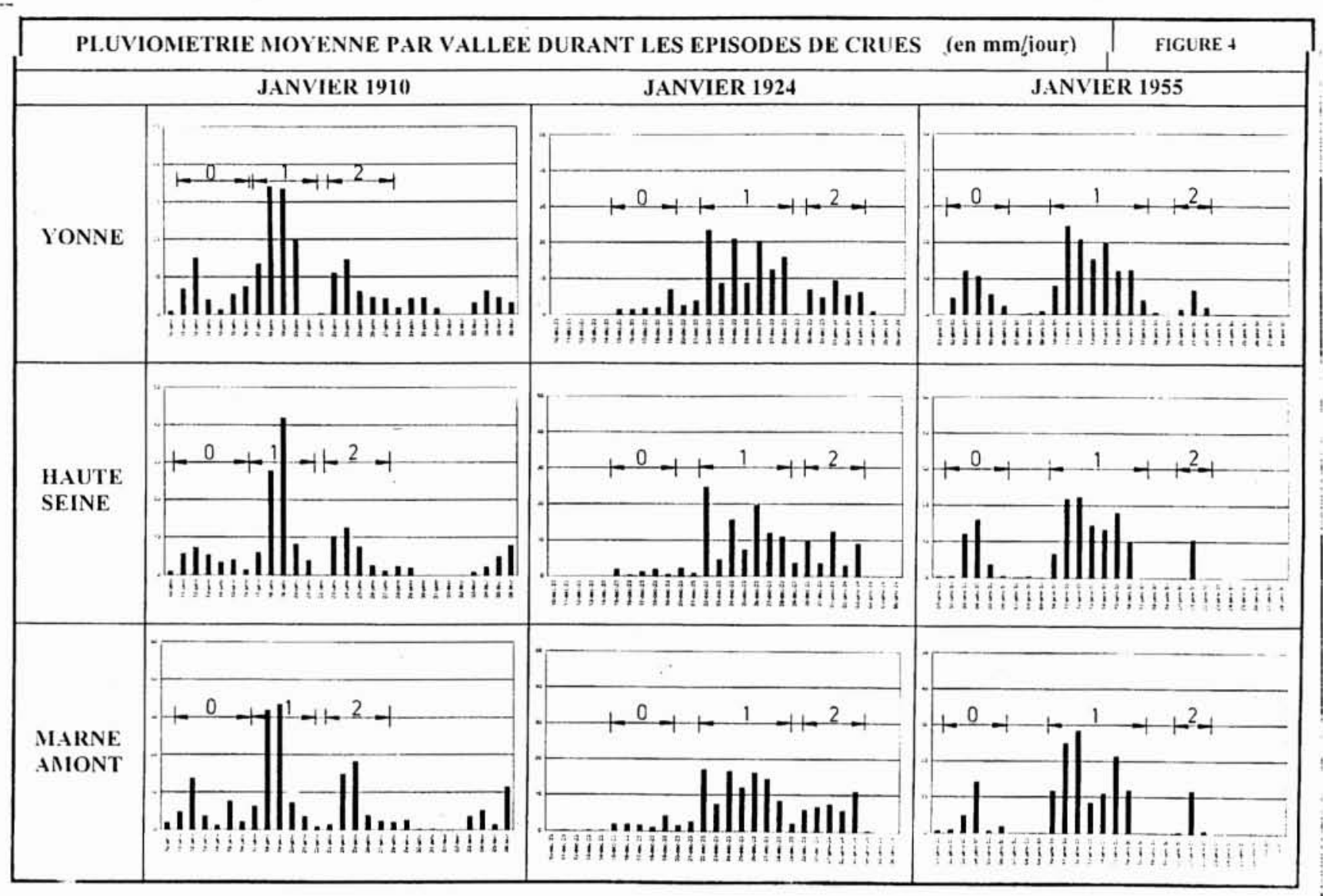

4. Pluviométrie moyenne par vallée durant les épisodes de crues (en $\mathrm{mm} / \mathrm{jour}$ ).

fait principalement des deux journées de précipitation intenses les 18 et 19 janvier.

- à Paris, le débit maximum exceptionnel de janvier 1910 résulte directement de la concomitance des ondes de crue de la Marne, de la Seine et de la seconde pointe de l'Yonne. Les deux crues de Janvier 1924 et 1955 affichent un débit similaire d'environ $2100 \mathrm{~m}^{3} / \mathrm{s}$ : l'apport un peu moins nourri de la Marne pour la crue de 1924 a été compensé par le soutien de la seconde pointe de l'Yonne.

Il est à noter que les niveaux d'eau maximum atteints sur la Seine aval ne reflètent pas totalement cette hiérarchie hydrométrique du fait des améliorations apportées aux écoulements du lit mineur entre 1910 et 1955.

Les figures 5 et 6 témoignent malgré tout de fortes similitudes entre les 3 crues tant au niveau de la pluviométrie durant l'épisode intense que de la forme générale des hydrogrammes à Paris.

\subsection{Précisions attachées aux débits de crue}

Les débits reportés figure 4 ont été testés à l'aide du modèle MRIF présenté dans un article d'accompagnement : la comparaison des lignes d'eau calculées et celles observées à l'époque avec ces débits, a permis de conforter la cohérence d'ensemble des résultats, lorsqu'on tient compte des travaux d'amélioration effectués depuis 1910. Les tests de sensibilité effectués tendent à justifier ces valeurs avec une fourchette d'incertitude de l'ordre de $50 \mathrm{~m}^{3} / \mathrm{s}$ pour l'ensemble des stations $\left( \pm 50 \mathrm{~m}^{3} / \mathrm{s} \Leftrightarrow \pm 15 \mathrm{~cm}\right.$ environ à Paris Austerlitz).

\subsection{Périodes de retour}

Le tableau 3 propose les valeurs de période de retour à associer aux débits maxima de crue aux principales stations.

Cette estimation est tirée d'une analyse statistique des débits maximum annuels naturels de crues reconstitués sur la période 1876-1995 [4]. Elle montre que la crue de janvier

Tableau 3. - Valeurs de période de retour à associer aux débits maxima.

\begin{tabular}{|c|c|c|c|c|}
\hline Station & Vallée & janvier 1910 & janvier 1924 & janvier 1955 \\
\hline Courlon & Yonne aval & 150 ans & 30 ans & 30 ans \\
Noisiel & Marne & 40 ans & 20 ans & 30 ans \\
Melun & Seine amont & 100 ans & 30 ans & 30 ans \\
Paris & Seine aval & 100 ans & 40 ans & 40 ans \\
Mantes & Seine aval & 100 ans & 30 ans & 30 ans \\
\hline
\end{tabular}




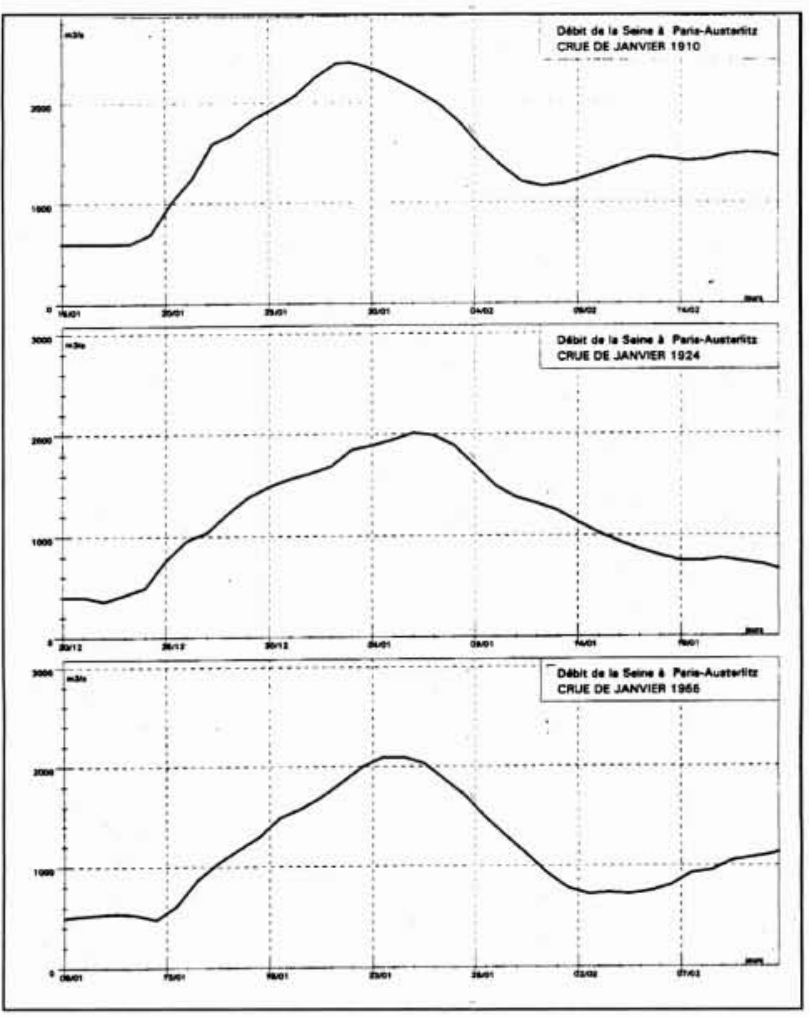

5. Hydrogrammes calculés à Paris pour les trois crues de 1910, 1924 et 1955.

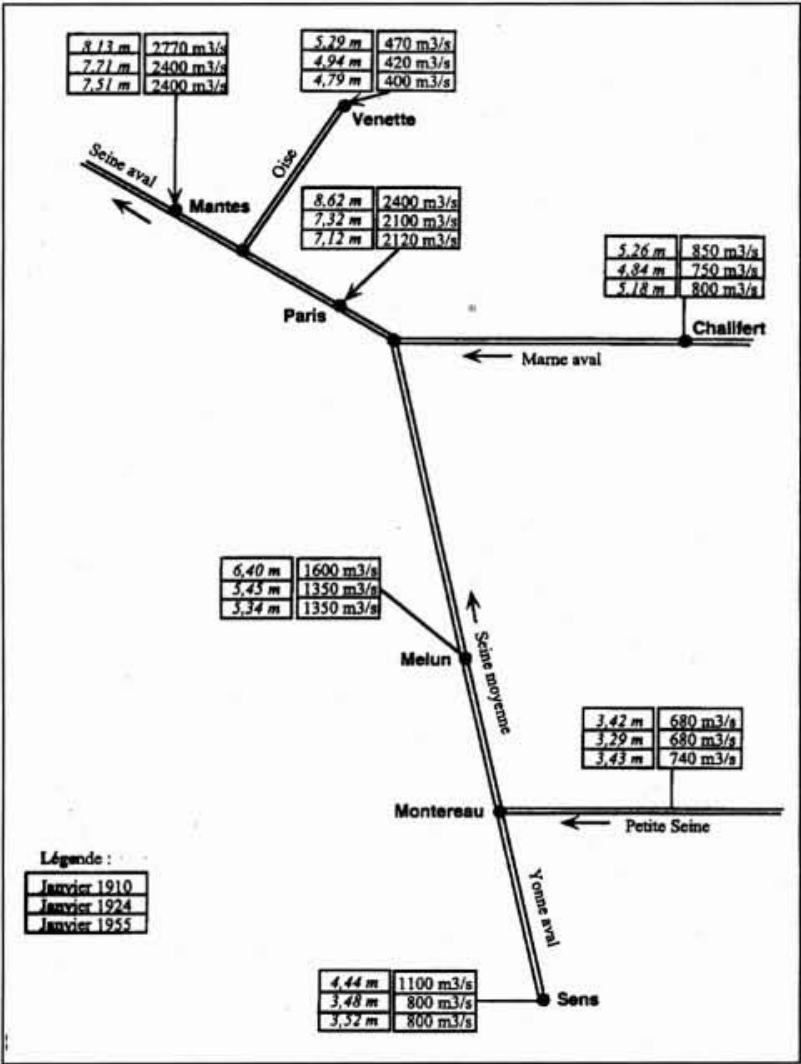

6. Hauteurs d'eau maximales relevées et débits de pointes reconstitués pour les trois crues de janvier 1910, janvier 1924, janvier 1955.
1910 est affectée d'une période de retour centennale ou supérieure pour toute les grandes vallées, à l'exception peut-être de la Marne où elle se démarque moins des autres crues de la période analysée.

En revanche les deux autres crues n'ont pas le même caractère exceptionnel, puisque leur période de retour ne dépasse pas 30 ans sur la plupart des vallées.

\section{V — SYNTHÈSE : TYPOLOGIE DES CRUES MARQUANTES DU BASSIN DE LA SEINE}

L'analyse des grandes crues du bassin de la Seine au cours de ce siècle conduit à dégager la typologie suivante.

\section{- 5.1 Crues simples}

Elles sont générées par un seul épisode pluvieux généralisé. La combinaison des 3 ondes de crues formées sur les bassins de l'Yonne, de la Haute Marne et de la Haute Seine peut engendrer une crue majeure sur la Seine aval en cas de crue majeure sur la Seine et la Marne. Ce fut le cas en 1955.

Le graphe 7 illustre la genèse de ce type de crue.

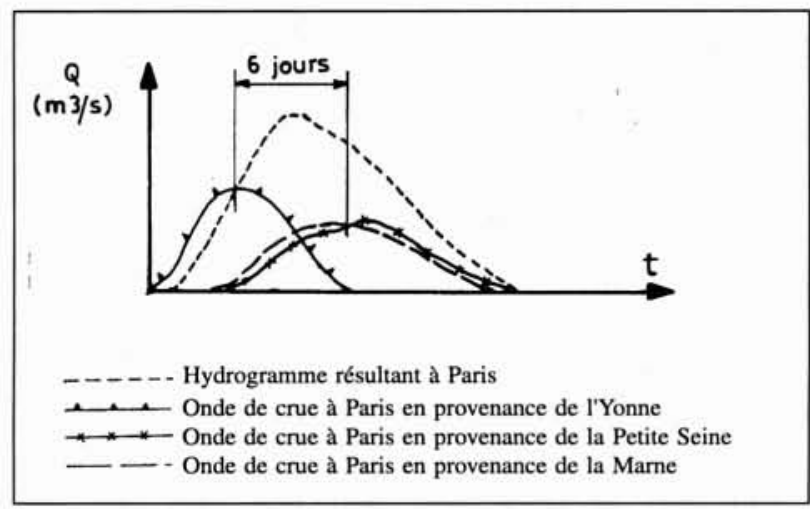

\section{Crue simple.}

\subsection{Crues doubles}

Il s'agit des crues générées par deux épisodes à intervalle rapproché.

Cette situation provoque une onde de crue unique sur la vallée de la Seine et de la Marne, mais deux ondes distinctes sur l'Yonne ; la seconde pointe coïncide à Paris avec celle en provenance de la Marne et de la Petite Seine, comme l'illustre le graphe 8 .

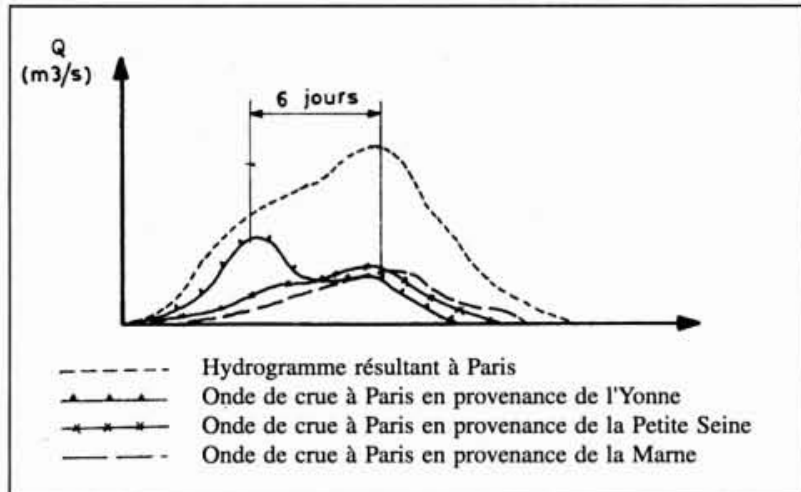

8. Crue double. 


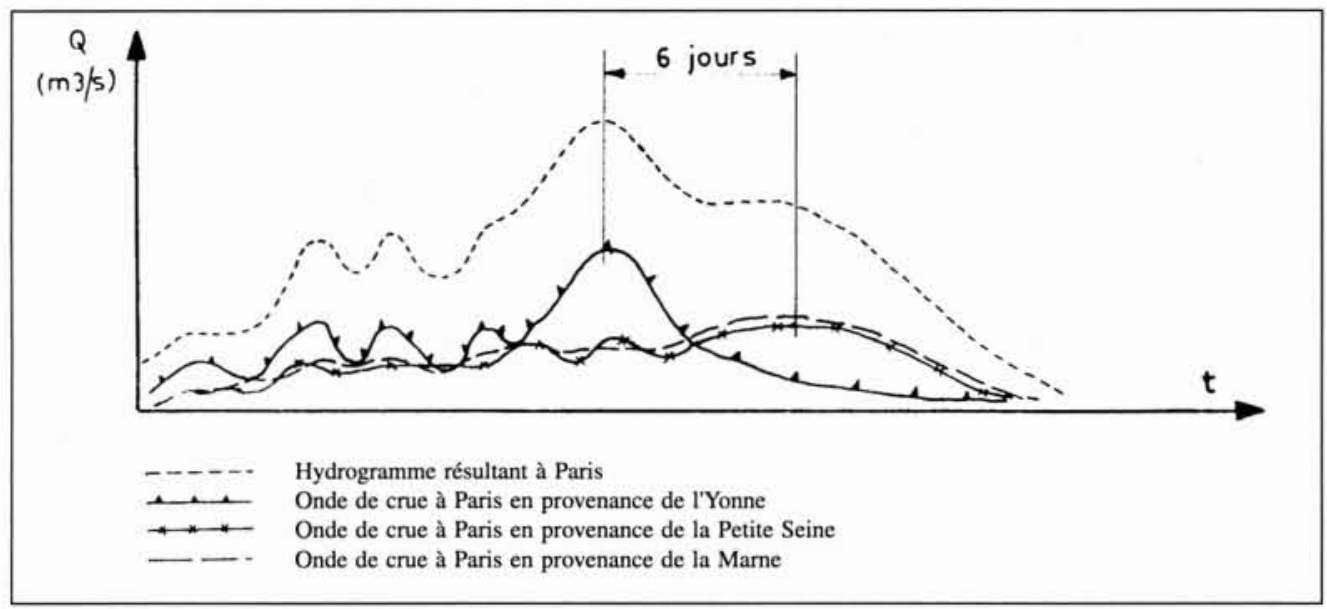

\section{Crue multiple.}

Les crues de janvier 1910 et janvier 1924 rentrent dans cette catégorie.

\section{- 5.3 Crues multiples}

Ce type correspond à une succession de petites crues générées par des pluies répétées pendant plusieurs semaines, suivies par un événement pluvieux plus important touchant particulièrement la vallée de l'Yonne. On assiste tout d'abord sur les vallées de la Seine et de la Marne à une montée régulière des eaux, les petites crues de l'Yonne se succédant les unes aux autres.

L'événement pluvieux génère ensuite une onde de crue marquée sur l'Yonne qui se superpose aux eaux déjà hautes de la Petite Seine et de la Marne et engendre ainsi une crue importante sur la Seine amont et à Paris, comme l'illustre le graphe 9.

L'événement de janvier 1982, dont le débit a atteint à Paris près de $1800 \mathrm{~m}^{3} / \mathrm{s}$, est représentatif de ce type de crue.

Notons en conclusion que les débits reconstitués ici sont significatifs d'une hydrologie naturelle quantifiable en terme de période de retour, qui découle directement du contexte climatique et géomorphologique du bassin de la Seine.

L'étape suivante consiste à évaluer le risque résiduel d'inondation induit par les événements historiques, en inté- grant l'ensemble des aménagements réalisés depuis 1910 sur le bassin de la Seine.

Ce vaste sujet fait appel à des outils de simulation hydraulique adaptés : il est abordé dans une autre communication consacrée au volet hydraulique.

\section{RÉFÉRENCES}

[1] : Notes internes du service hydrométrique du bassin de la Seine. (Archives DIREN - Juil 1910)

[2] : Evaluation des impacts socio-économiques des crues en Région llede-France - 1.Hydrologie - Annexe 1.2: Constitution de fonctions de transfert pluie efficace - débits. (Hydratec - Mai 1997)

[3] : Evaluation des impacts socio-économiques des crues en Région llede-France - 1.Hydrologie - Annexe 1.1 : Reconstitution des débits de crue du bassin de la Seine. (Hydratec - Mai 1997)

[4] : Evaluation des impacts socio-économiques des crues en Région Ilede-France - 1. Hydrologie - Volet A : analyse statistique des débits naturels de crues reconstituées sur la période 1876 - 1995 (Hydratec - Mai 1997)

[5] : Résultats de jaugeages réalisés sur la Seine aval durant la période de Janvier à Mars 1910. (Willemin - SNS - Archives DIREN - Mai 1910).

[6] : Evaluation des impacts socio-économiques des crues en Région Ilede-France - 1.Hydrologie - Annexe 1.3.2 : Reconstitution de l'hydrologie de la crue de Janvier 1924. (Hydratec - Mai 1997) 